\title{
How occasional backstepping can speed up a processive motor protein
}

\author{
Martin Bier ${ }^{\mathrm{a}, *}$, Francisco J. Cao ${ }^{\mathrm{b}, \mathrm{c}}$ \\ a Department of Physics, East Carolina University, Greenville, NC 27858, USA \\ b Departamento de Física Atómica, Molecular y Nuclear, Universidad Complutense de Madrid, Avenida Complutense s/n, 28040 Madrid, Spain \\ c Observatoire de Paris, LERMA, Laboratoire Associé au CNRS UMR 8112, 61, Avenue de l'Observatoire, 75014 Paris, France
}

\section{A R T I C L E I N F O}

\section{Article history:}

Received 31 July 2010

Accepted 8 November 2010

\section{PACS:}

05.40.-a

87.16.Nn

Keywords:

Molecular motors

Kinesin

Backstepping

Entropy

Activation state

\begin{abstract}
A B S T R A C T
Fueled by the hydrolysis of ATP, the motor protein kinesin literally walks on two legs along the biopolymer microtubule. The number of accidental backsteps that kinesin takes appears to be much larger than what one would expect given the amount of free energy that ATP hydrolysis makes available. This indicates that backsteps are not simply the forward stepping cycle run backwards. We propose here a simple effective model that consistently includes the backstep transition. Using this model, we show how more backstepping increases the entropy of the final state, and probably also the activation state, thus reducing their free energy. This free energy reduction of the activation state (related to backstepping) speeds up the catalytic cycle of the kinesin, making both forward and backward steps more frequent. As a consequence, maximal net forward speed is achieved at nonzero backstep percentage. In addition, the optimal backstep percentage coincides with the backstep percentage measured for kinesin. This result suggests that, through natural selection, kinesin could have evolved to maximal speed.
\end{abstract}

(C) 2010 Elsevier Ireland Ltd. All rights reserved.

\section{Introduction}

Processive motor proteins are among the tiniest engines known to man. These proteins utilize the energy of ATP hydrolysis to literally walk along a biopolymer (Howard, 2001). In a living cell they help maintain organization by transporting cargo, like organelles or vesicles filled with chemicals.

Already one and a half decade ago the stepping of the processive motor protein kinesin was made visible on the nanometer scale with optical tweezers (Howard, 2001). Early communications (Visscher et al., 1999; Schnitzer et al., 2000) reported that 5-10\% of all steps of kinesin were backward. But smaller fractions were described later on as methods and materials improved and better resolutions were achieved; Nishiyama et al. (2002) gave $1 / 220$ and Carter and Cross (2005) gave 1/802. Theoreticians have always been interested in backstep fractions as they can help verify stochastic models.

In this article we will show how, in the Brownian environment of the motor protein, a "well-tuned" backstep fraction can actually help the motor speed up. We will show how the backstep fraction that leads to the highest net speed can be evaluated and how the resulting expression contains no freely adjustable parameters.

\footnotetext{
* Corresponding author at: Department of Physics, East Carolina University, Greenville, NC 27858, USA. Tel.: +252 328 6428; fax: +252 3286314 .

E-mail address: bierm@ecu.edu (M. Bier).
}

Finally, we will see how the experimentally established backstep fraction of kinesin is close to our predicted optimal backstep fraction.

\section{An appropriate model for kinesin}

The operation of an ion pump is generally modelled with a cycle as depicted in Fig. 1. At equilibrium the product of the forward rates, $k_{12} \times k_{23} \times \cdots \times k_{n 1}$, equals the product of the backward rates, $k_{21} \times k_{32} \times \cdots \times k_{1 n}$, and no net cycling occurs. To drive the protein through the sequence of states, $S_{1}, S_{2}, \ldots, S_{n}$, a driving energy is necessary (Hill, 1968). Such energy comes available if one of the steps involves the binding of ATP and if the protein, in subsequent steps, catalyzes the hydrolysis of the bound ATP. Eventually the remaining ADP and an inorganic phosphate have to be released so as to complete the cycle and to put the protein again in a state in which it can bind a new ATP. Under physiological conditions the hydrolysis of ATP makes $G_{\text {ATP }}=22 k_{B} T$ units of free energy available. In the course of a cycle of a membrane pump like Na,K-ATPase, part of $G_{\text {ATP }}$ is utilized to bind, transport and release on the other side of the membrane three sodium ions and two potassium ions. The transport is generally against the electrochemical potential of the involved ions. Consistent with the model of Fig. 1, it is found that with a sufficiently low ATP-ADP potential and a high electrochemical potential for sodium and potassium the operation of the pump can be reversed (Läuger, 1991). 


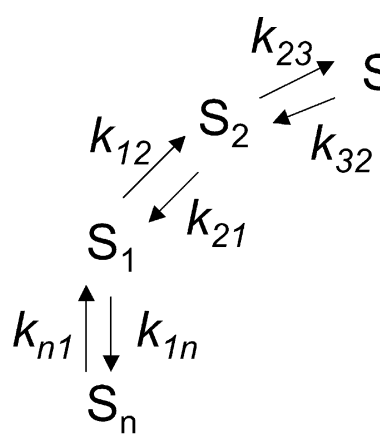

$\mathrm{S}_{5}$

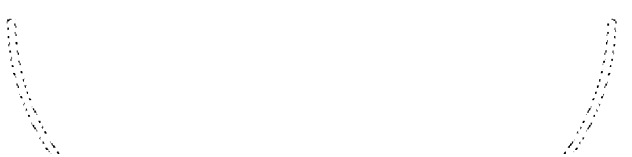

Fig. 1. An abstract conception of kinesin's catalytic cycle. The cycle involves the binding and hydrolysis of ATP, the actual mechanical stepping, and the release of ADP and inorganic phosphate. Each reaction is driven by an energy difference $G_{i, i+1}$ and the ratio of the forward and backward transition rate is an exponential function of $G_{i, i+1}$.

The stepping kinesin appears to operate in a fundamentally different way. Tight coupling, i.e. an $8 \mathrm{~nm}$ step for every hydrolyzed ATP and a hydrolyzed ATP for every $8 \mathrm{~nm}$ step, has been observed for kinesin (Schnitzer and Block, 1997; Hua et al., 1997). Without a mechanical load it is just the $G_{\text {ATP }}$ that is driving the cycle in Fig. 1. Every mechanical step should correspond to one revolution around the catalytic cycle. If a backstep would correspond to the cycle being run in the direction against the ATP hydrolysis, then we should have $p_{b} / p_{f}=\exp \left[-G_{\text {ATP }}\right]$ for the ratio of the backstep probability and the forward step probability. However, exp[-22] turns out 7 orders of magnitude smaller than the measured backstep fractions that were mentioned before. Furthermore, a model like in Fig. 1 leads to a stopping force, i.e. the load at which the kinesin comes to a standstill, that is determined by $G_{\text {ATP }}=F_{\text {st }} L$, where $L$ is the steplength. If $G_{\mathrm{ATP}}=F_{\mathrm{st}} L$, then the two forces, chemical and mechanical, that are driving the cycle in opposite directions cancel each other out. But, with $G_{\text {ATP }}=22$ and $L=8 \mathrm{~nm}$, the equation $G_{\mathrm{ATP}}=F_{\mathrm{st}} L$ predicts a stopping force $F_{\mathrm{st}}$ that is about twice as large as the measured $7 \mathrm{pN}$ (Visscher et al., 1999; Schnitzer et al., 2000; Carter and Cross, 2005). Most importantly, it appears that kinesin still hydrolyzes ATP when it is pulled back with the stopping force and even when it is made to walk backwards with a load larger than the stopping force (Carter and Cross, 2005; Molloy and Schmitz, 2005). All these observations make a model as depicted in Fig. 1 untenable.

We are thus led to a different model for the stepping motor protein (Bier, 2003). After the detachment of the back leg, the attached leg reorients and brings the detached leg to the vicinity of the next forward binding site (see Fig. 2). Brownian motion is supposed to eventually make the detached leg hit the next forward site. Attachment there can then occur. The trailing leg next detaches, thus completing a forward step. It is the energy $G$ that drives the reorientation of the attached leg and so biases the Brownian step towards the forward site. That reorientation is only one transition in the entire hydrolysis cycle of the kinesin. Therefore, the reorientation energy $G$ is smaller than $G_{\text {ATP. }}$. For the backward binding probability $p_{b}$ and the forward binding probability $p_{f}$ we can thus have $p_{b} / p_{f} \gg \exp \left[-G_{\text {ATP }}\right]$ (Bier, 2007). Assuming that binding to the backward site is followed by a backstep, the model of Fig. 2 can actually lead to an accurate accounting for the backstep rates (Bier, 2003; Bier, 2007). Ultimately, the scheme depicted in Fig. 3 is a more appropriate model for the Brownian stepper than the kinesin's catalytic cycle of Fig. 1 alone. In

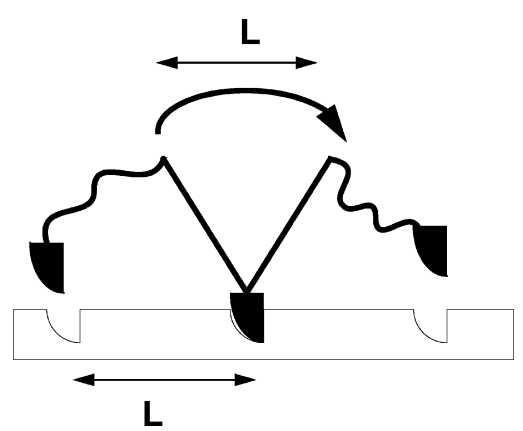

Fig. 2. The Brownian step of a processive motor protein. After detachment of the trailing leg, the attached leg reorients and brings the detached leg to the vicinity of the next forward binding site. After that, random diffusive motion will usually make the detached leg hit and attach to the forward binding site. Next the trailing leg detaches and a forward step is thus completed. However, there is a nonzero probability that the detached leg hits and attaches to the backward binding site. We assume that such backward site binding is what triggers the backstep. The probabilities $p_{f}$ and $p_{b}$ for forward and backward binding depend on the energy behind the reorientation, the applied load force, and, as the process occurs in the Brownian regime, on $k_{B} T$, the natural unit of thermal energy.

the scheme in Fig. 3 at a particular point in the ATP hydrolysis cycle a kind of coin-toss takes place and the forward-backward determination occurs. The corresponding mechanical steps run in a dimension that is perpendicular to the plane of the chemical cycle.

\section{Thermodynamics of backstepping and speed optimization}

The question that needs answering at this point is: why has natural selection led to a backstep probability that is many orders of magnitude larger than the bare minimum of exp[-22] that thermodynamics requires? As was mentioned before, kinesin pulls organelles or chemical-filled vesicles across a eukaryotic cell. The reason that eukaryotic cells have such an active transport system in the first place is that they are, unlike prokaryotic cells, too large to rely on diffusion for their transport needs. Kinesin's stepping speed ultimately determines how fast a eukaryotic cell can react to environmental stimuli. There should be selectional advantage in engaging a kinesin that runs faster.

Our claim is that the entropy increase due to the forward versus backward "choice" can provide an answer to the question. The free energy $k_{B} T \ln 2$ that is associated with a doubling of the avail-

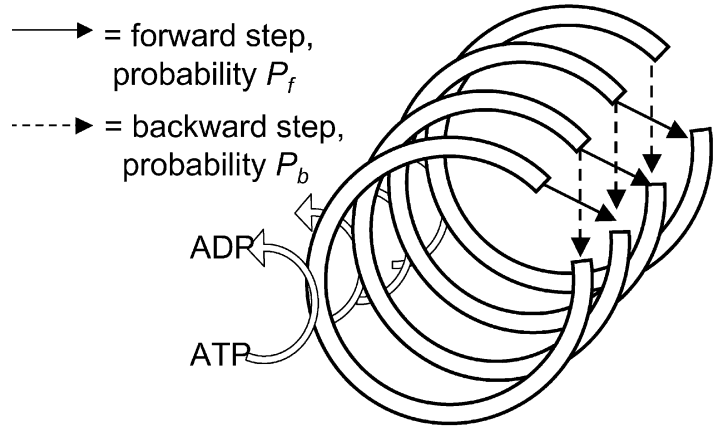

Fig. 3. Kinesin still hydrolyzes ATP when it is pulled back with a force larger than the stopping force. A setup like Fig. 1, with the ATP-ADP potential and the mechanical load pushing a single cycle in opposite directions, is therefore not the appropriate model and needs modification. Here we let the mechanical dimension run perpendicularly to the plane of the chemical cycle. ATP hydrolysis drives the chemical cycle in a clockwise direction. At the mechanical junction (cf. Fig. 2) a forward versus backward "decision" is made. 
able space is still small relative to the $22 k_{B} T$-units released by ATP hydrolysis, but, we will argue, it can play a role in the optimization of a processive motor protein.

Imagine a large number, $N$, of motor proteins that are all at the same position. There is only one possible microstate for this macrostate. Next, let all of these $N$ motors take a step. If all of these motors step forward, we will continue to have only one microstate for the macrostate of the system. But if, on the other hand, we allow for one backstep in the $N$ steps, then there are $N$ possible microstates. This is because the backstep can occur with any of the $N$ motors. The increase in the number of microstates implies an increase in entropy. A more complete and precise way to think of this is as follows. Before a step occurs, the entropy is $S_{0}=k_{B} \sum_{i} p_{0}(i) \ln p_{0}(i)$, where $p_{0}(i)$ is the probability of the microstate $i$. After one catalytic cycle and its accompanying step, the probabilities of the microstates in the forward site are $p_{1}(i, f)$ while for those in the backward site are $p_{1}(i, b)$. This makes that the entropy after the step is $S_{1}=k_{B} \sum_{i} \sum_{l=b f} p_{1}(i, l) \ln p_{1}(i, l)$. As after one cycle the macrostate of the motor is recovered, but one site ahead or behind, these probabilities are related to those before the step by $p_{1}(i, f)=p_{0}(i) p_{f}$ and $p_{1}(i, b)=p_{0}(i) p_{b}$. Therefore, the entropy increase in one step is $\Delta S=S_{1}-S_{0}=-k_{B}\left(p_{f} \ln p_{f}+p_{b} \ln p_{b}\right)$, i.e. it is due solely to the increase in the "position entropy" - given by the probability spreading of the motor position among sites (Leff, 2003).

With a forward step probability $p_{f}$ and a backstep probability $p_{b}$ we have for the speed of the stepper $v \propto p_{f}-p_{b}=1-2 p_{b}$. So the direct mechanical effect of an increase of the backstepping rate is a decrease of the speed, i.e. $\delta v \propto-2 \delta p_{b}$. But the backstep probability $p_{b}$ also increases the entropy of the final state. This entropy increase implies a decrease in the free energy of the final state making an additional amount of free energy available for the process to the amount of:

$\tilde{G}=T \Delta S=-\left(p_{f} \ln p_{f}+p_{b} \ln p_{b}\right)$.

Here $\tilde{G}$ is expressed in units of $k_{B} T . k_{B} T$ can be thought of as the natural unit of thermal energy and we will express energy in $k_{B} T$ units throughout the remainder of this article. As $p_{b}$ is observed to be small $\left(p_{b}<0.01\right)$, first order approximations will be sufficiently accurate and the theory we develop in this article is a first order theory in $p_{b}$. At first order in $p_{b}$, Eq. (1) reduces to $\tilde{G} \approx p_{b}\left(1-\ln p_{b}\right)$. So a variation $\delta p_{b}$ in $p_{b}$ leads to a variation

$\delta \tilde{G} \approx-\left(\ln p_{b}\right) \delta p_{b}$

in the free energy that becomes available.

We contend that the $\tilde{G}$ of Eq. (1) can be utilized to speed up the catalytic cycle of the motor protein. Throughout biology, energy is used to build and maintain ordered, low entropy structures. The sodium-glucose cotransporter concentrates glucose inside a cell. It does so by using the energy of a cotransported sodium ion. Entropic energy, in turn, is utilized whenever a transporter exploits a concentration gradient as a source of energy. In this way there are many examples of biomolecules that use the generation of entropy as a source of free energy. In the course of its cycle the $\mathrm{Na}, \mathrm{K}, 2 \mathrm{Cl}$-cotransporter brings one sodium ion, one potassium ion, and two chloride ions from the outside to the inside of a cell. The cycle is electroneutral. Under physiological conditions, this cotransporter effectively uses the transmembrane concentration gradients for sodium and chloride as an energy source to accumulate potassium inside the cell. More in general, the Nernst potential $V=R T \ln \left(C_{\text {in }} / C_{\text {out }}\right)$ for an ion or molecule is due to a difference between the concentrations inside $\left(C_{\text {in }}\right)$ and outside $\left(C_{\text {out }}\right)$ a cell. The Nernst potential is purely entropic in origin and it is a commonly utilized source of energy in a living cell.
We next need to quantify how a $\delta \tilde{G}$ (cf. Eq. (2)) can speed up a catalytic cycle. To this end we return to the catalytic cycle depicted in Fig. 1. The chemical part of kinesin's catalytic cycle (the binding of ATP, release of ADP and inorganic phosphate, detachment and attachment of the legs to the microtubule) is well modelled by a setup like Fig. 1 . With energy expressed in units of $k_{B} T$, the energy that drives the transition from $S_{i}$ to $S_{i+1}$ is $G_{i, i+1}$ and we have $\exp \left[G_{i, i+1}\right]=k_{i, i+1} / k_{i+1, i}\left(\right.$ Howard, 2001). Adding $\delta \tilde{G}$ to $G_{i, i+1}$ we have

$e^{G_{i, i+1}+\delta \tilde{G}}=\frac{k_{i, i+1}+\delta k_{i, i+1}}{k_{i+1, i}+\delta k_{i+1, i}}$.

The apportionment factor $\alpha$ parameterizes how much of the change $\delta \tilde{G}$ goes towards increasing the forward rate $k_{i, i+1}$, and how much towards decreasing the backwards rate $k_{i+1, i}$,

$k_{i, i+1}+\delta k_{i, i+1}=k_{i, i+1} e^{\alpha \delta \tilde{G}}$,

$k_{i+1, i}+\delta k_{i+1, i}=k_{i+1, i} e^{-(1-\alpha) \delta \tilde{G}}$.

We consider here the case $\alpha \approx 1$, in which the main effect is an increase of the forward rate. For small $\delta \tilde{G}$ we have $\exp [\delta \tilde{G}] \approx 1+\delta \tilde{G}$, which in the case $\alpha \approx 1$ leads to

$\delta \tilde{G} \approx \frac{\delta k_{i, i+1}}{k_{i, i+1}}$.

So, ultimately, the relative increase of the forward rate $k_{i, i+1}$ is just $\delta \tilde{G}$. It is also possible to consider the case $\alpha \approx 0$, when $\delta \tilde{G}$ goes towards reducing the reverse rate $k_{i+1, i}$. However, when a reaction is already reasonably irreversible (i.e. $k_{i, i+1} / k_{i+1, i}$ being sufficiently large) this is not an efficient way to speed up the reaction. We thus consider here that the $\delta \tilde{G}$ brings down the energy of the product state and the energy of the activation barrier by the same amount. This would happen if the transition state (the one that represents being on the top of the activation barrier) already has microstates associated with the forward and backward possibilities. In this case, the effect in the transition state is the same as in the product state and the increase in "position entropy" also lowers the energy of the activation barrier by the same amount. In the following we will ignore $k_{i+1, i}$ and write $k_{i}$ for $k_{i, i+1}$.

The energies associated with the measured $p_{b}$ 's are small. For $p_{b}=1 / 802$, Eq. ( 1 ) gives $\tilde{G}=0.01 k_{B} T$-units. Following Eq. (6) we see that this translates into a $1 \%$ change in the speed of a transition relative to $p_{b}=0$ case.

When one transition in a cycle like in Fig. 1 is speeded up by $1 \%$, it does not imply that the time to go through the entire cycle will decrease by $1 \%$. That latter change will generally be less than $1 \%$. We let $C_{k_{i}}^{v}$ be a so-called "control coefficient" (Kacser and Burns, 1973; Heinrich and Rapoport, 1974); this is a dimensionless parameter that puts a figure on the influence that the rate $k_{i}$ of transition $i$ has on the rate $v$ of going through the entire cycle. This cycling rate is also the stepping speed of the motor protein. We take

$C_{k_{i}}^{v}=\frac{\delta v / v}{\delta k_{i} / k_{i}}$.

Going to the limit $\delta k_{i} \rightarrow 0$, we see that the control coefficient is essentially a $\log$ arithmic derivative, i.e. $(\partial \ln v) /\left(\partial \ln k_{i}\right)$. So suppose $k_{i}$ is changed by $1 \%$. The control coefficient $C_{k_{i}}^{v}$ then gives roughly the percentage by which $v$ changes as a result. Assume that you change all of the transition rates in the cycle with the same percentage. This is like scaling the time and, obviously, the speed $v$ would be changed by the same percentage. This leads to the identity $\sum_{i=1}^{n} C_{k_{i}}^{v}=1$, which is commonly known as the Summation Theorem (Kacser and Burns, 1973; Heinrich and Rapoport, 1974). If $k_{i}$ represents the rate limiting transition, then $C_{k_{i}}^{v}$ will be close to unity. $C_{k_{i}}^{v}$ will be close to zero if the $k_{i}$-transition is very fast compared to other transitions in the cycle. Generally, the control coefficient $C_{k_{i}}^{v}$ 
will be a positive number between zero and unity that expresses the amount of control that transition $i$ has over the speed of the entire cycle. In most biochemical networks and protein catalysis cycles there is no single rate limiting step. Instead, control is somewhat equally distributed over several steps.

Since $\delta \tilde{G}$ gives the relative change of $k_{i}$, we have for the speed of the motor protein and its increase due to the addition of $\delta \tilde{G}$ to step $i$

$v+\delta v \propto 1+C_{k_{i}}^{v} \delta \tilde{G} \approx 1-C_{k_{i}}^{v}\left(\ln p_{b}\right) \delta p_{b}$.

We combine this result with the mechanical effect of the backstepping, which, we saw earlier, was described by $v \propto 1-2 p_{b}$ or, consequently, $\delta v \propto-2 \delta p_{b}$. We now have for the net variation of $v$ when $p_{b}$ is varied:

$\delta v \propto-\left\{2+C_{k_{i}}^{v} \ln p_{b}\right\} \delta p_{b}$.

The speed $v$ has a maximum when the variation $\delta v$ equals zero, i.e.

$\ln p_{b}=-\frac{2}{C_{k_{i}}^{v}}$.

This equation relates the optimal backstepping fraction to the control coefficient of the transition that includes the free energy derived from the forward-versus-backward entropy. The $p_{b}=1 / 220$ from Nishiyama et al. (2002) leads to $C_{k_{i}}^{v}=0.4$. The $p_{b}=1 / 802$ from Carter and Cross (2005) leads to $C_{k_{i}}^{v}=0.3$.

Under physiological conditions the transitions in the cycle of a motor protein are driven by energies of about $2 k_{B} T$-units (Cross, 2004; Bier, 2007). Consequently, reverse transitions in the catalytic cycle are relatively rare. If we ignore the backward rates in the cycle of the motor protein, then the control coefficient $C_{k_{i}}^{v}$ is simply the fraction of time during the cycle that the motor protein spends in state $S_{i}$. That time equals $1 / k_{i, i+1}$. When the back leg of kinesin detaches, the hydrolysis of the ATP bound to that leg proceeds concurrently. The release of the ADP is supposed to take place upon rebinding of the detached leg to the microtubule. In a comprehensive review (Cross, 2004) a rate of $\sim 250 \mathrm{~s}^{-1}$ is given for the back-leg-detachment/hydrolysis transition. With the stepping rate of $\sim 100 \mathrm{~s}^{-1}$, we are indeed led to a control coefficient of 0.4 for this transition. The same reference lists $\sim 300 \mathrm{~s}^{-1}$ as the rate for the ADP release transition. This would correspond to a control coefficient of about 0.3. With Eq. (10) we find that control coefficients between 0.3 and 0.4 lead to backstep rates between $1 / 150$ and $1 / 800$. We infer that observed control coefficients then predict the correct order of magnitude for the observed backstep rates of Nishiyama et al. (2002) and Carter and Cross (2005). Eq. (10) and its underlying hypothesis are thus found in agreement with experimental reality.

\section{Discussion}

There is no detailed knowledge of how kinesin's internal structure changes as it goes through the cycle of conformational transitions. We can therefore not point at a specific biomolecular mechanism for the utilization of the free energy that is made available by a slightly increased $p_{b}$. It is conceivable that, at some point in the cycle, the position of a cluster of atoms within the kinesin determines whether a step will go forward or backward. We can depict the corresponding reaction coordinate as the double well in Fig. 4. When the cluster is in the left well, a forward step is made. When the cluster is in the right well, a backstep occurs. The activation barrier between the states in Fig. 4 needs to be sufficiently low for quick equilibration to take place. A Boltzmann distribution between the two wells then determines the forward-versus-backward step probability. With an energy difference of $\varepsilon$ between the wells we

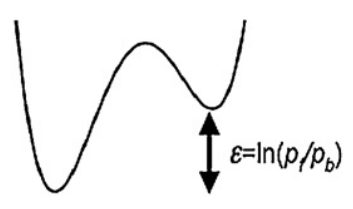

Fig. 4. A model for the mechanism behind the backstepping. Whether a step goes forward or backward depends on the position of a cluster within the processive motor protein. The corresponding reaction coordinate for the cluster is depicted. The left and right well stand for forward and backward step, respectively. Eliminating the backstepping would mean eliminating the right well, thus reducing the number of available states and the entropy. The energy difference $\varepsilon$ between the wells is related to the forward-versus-backward stepping probability.

have $\varepsilon=\ln \left(p_{f} / p_{b}\right)$. For kinesin, with a backstep fraction of $1 / 802$, the energy difference is $\varepsilon \approx 7 k_{B} T$-units.

It is generally true that having more configurations available within a macrostate effectively lowers the free energy of that state. An example illustrates how this is often important on the level of a single biomolecule. For a protein there are many ways to be unfolded and randomly coiled, i.e. there are many, say $\Omega_{u}$, unfolded microstates. The number of microstates, $\Omega_{f}$, for the correctly folded state is generally orders of magnitude smaller (Jackson, 2006). So the folding of a protein actually requires a free energy $T \Delta S=k_{B} T \ln \left(\Omega_{u} / \Omega_{f}\right)$. By making the folded state more flexible, i.e. by giving it more "statistical wiggle room," this required free energy may be brought down and that may lead to faster folding. To a large extent, the situation is analogous for the processive motor protein. By putting more available microstates at the end of the ATP hydrolysis cycle the motor can increase the free energy that is available to drive the step. Molecular dynamics simulations have been widely used to shed light on the dynamics of protein folding. They may become similarly illuminating for the case of stepping motor proteins.

What we have shown in this article is that for kinesin the measured percentage of backsteps and the measured rates for the conformational changes agree with the idea of occasional backstepping as a source of energy and a way to speed up the motor protein. It appears that kinesin operates very close to the optimum that we calculated. So, even within the $1 \%$ available margin, natural selection may have manipulated the backstepping rate to maximize kinesin's speed, because even small selective advantages could be fixed by natural selection (Futuyma, 1998; Padian, 2008; Kirschvink, 2000).

\section{Acknowledgments}

We are grateful to various people from the Humboldt Universität in Berlin for critical feedback during two seminars there. Fig. 3 was an idea of Wolfram Liebermeister. FJC acknowledges financial support from the MEC (Spain) through Research Project FIS2006-05895, and from UCM and CM (Spain) through Grant Nos. CCG07-UCM/ESP-2925 and UCM-BSCH GR58/08 920911.

\section{References}

Bier, M., 2003. Phys. Rev. Lett. 91, 1481041.

Bier, M., 2007. BioSystems 88, 301.

Carter, N.J., Cross, R.A., 2005. Nature 435, 308.

Cross, R.A., 2004. Trends Biochem. Sci. 29, 301.

Futuyma, D.J., 1998. Evolutionary Biology. Sinauer Associates Inc., Sunderland, MA, p. 377.

Heinrich, R., Rapoport, T.A., 1974. Eur. J. Biochem. 42, 89.

Hill, T.L., 1968. Thermodynamics for Chemists and Biologists. Addison-Wesley, Reading, MA.

Howard, J., 2001. Mechanics of Motor Proteins and the Cytoskeleton. Sinauer Associates, Sunderland, MA.

Hua, W., Young, E.C., Fleming, M.L., Gelles, J., 1997. Nature 388, 390.

Jackson, M.B., 2006. Molecular and Cellular Biophysics. Cambridge University Press, Cambridge, UK, pp. 71-86. 
Kacser, H., Burns, J.A., 1973. Symp. Soc. Exp. Biol. 27, 65.

Kirschvink, J.L., 2000. Bull. Seism. Soc. Am. 90, 312-323.

Läuger, P., 1991. Electrogenic Ion Pumps. Sinauer Associates Inc., Sunderland, MA.

Leff, H.S., 2003. In: Rex, A.F. (Ed.), Maxwell's Demon 2 - Classical and Quantum Information, Computing. Institute of Physics Publishing, Bristol, UK. Molloy, J.E., Schmitz, S., 2005. Nature 435, 285.
Nishiyama, M., Higuchi, H. Yanagida, T., 2002. Nat. Cell Biol. 4, 790.

Padian, K., 2008. Nature 451, 632-634.

Schnitzer, M.J., Visscher, K., Block, S.M., 2000. Nat. Cell Biol. 2, 718.

Schnitzer, M.J., Block, S.M., 1997. Nature 388, 386.

Visscher, K., Schnitzer, M.J., Block, S.M., 1999. Nature 400, 184. 